News

\title{
Fibromyalgia/ chronic fatigue (pain in muscles with tender points all over the body) - find out why?
}

Volume 4 Issue 3 - 2016

Mohamed M Hassan

President at Dr. Hassan Chiropractic \& Natural healing, USA

Correspondence: Mohamed M Hassan, President at Dr. Hassan Chiropractic \& Natural healing, Texas Chiropractic College, USA, Email dr.mmhassan@yahoo.com

Received: January 06, 2016 | Published: February 10, 2016 b) Chronic disorder associated with pain of the muscles and related soft tissue such as ligaments and tendons where pain is generally present for at least three months.

c) Key Characteristics: Is Muscle pain, Key aspect of Fibromyalgia is the presence of "tender points",

*Symptoms: Chronic pain is the most common. In most people it affects the entire body, although it may start in one area such as the neck and shoulders, and spread to other areas over a period of time.

Symptoms: It may range from mild discomfort to pain severe enough to limit work, social activities, and everyday tasks.

Symptoms of Fibromyalgia can be associated with:

a. Headaches, especially tension and migraine headaches.

b. Abdominal pain, bloating, alternating constipation.

c. Urinary bladder spasms and irritability may cause urinary urgency or frequency.

d. Changes in skin color: Due to more sensitivity of skin blood circulation to temperature changes,

e. Chronic fatigue: To the point that making simple daily activities almost impossible.
IF You (Loved one or someone you know) may be experiencing moderate or severe fatigue with a lack of energy, decreased exercise endurance, or the kind of exhaustion that results from the flu or lack of sleep. They may be suffering from Fibromyalgia: that kept them from participating in their life or Simple daily activities.

\section{Acknowledgments}

None.

\section{Conflicts of Interset}

None. 